Research Article

\title{
Optimal Stable Approximation for the Cauchy Problem for Laplace Equation
}

\author{
Hongfang Li and Feng Zhou \\ College of Science, China University of Petroleum (East China), Qingdao 266580, China \\ Correspondence should be addressed to Hongfang Li; sdongbb@163.com
}

Received 23 March 2016; Accepted 17 May 2016

Academic Editor: Ricardo Weder

Copyright (C) 2016 H. Li and F. Zhou. This is an open access article distributed under the Creative Commons Attribution License, which permits unrestricted use, distribution, and reproduction in any medium, provided the original work is properly cited.

\begin{abstract}
Cauchy problem for Laplace equation in a strip is considered. The optimal error bounds between the exact solution and its regularized approximation are given, which depend on the noise level either in a Hölder continuous way or in a logarithmic continuous way. We also provide two special regularization methods, that is, the generalized Tikhonov regularization and the generalized singular value decomposition, which realize the optimal error bounds.
\end{abstract}

\section{Introduction}

The Cauchy problem for the Laplace equation in particular, and for other elliptic equations in general, occurs in the study of many practical problems in areas such as plasma physics [1], electrocardiology $[2,3]$, bioelectric field problems [4], nondestructive testing [5], magnetic recording [6], and the Cauchy problem for elliptic equations $[7,8]$. These problems are known to be severely ill-posed [9], in the sense that the solution, if it exists, does not depend continuously on the Cauchy data in some natural norm (see, e.g., [5] and references therein). This is because the Cauchy problem is an initial value problem which represents a transient phenomenon in a time-like variable while elliptic equation describes steady-state processes in physical fields. A small perturbation in the Cauchy data, therefore, can affect the solution largely.

In this paper, we will concretely consider the following Cauchy problem for Laplace equation in a strip:

$$
\begin{aligned}
u_{x x}+u_{y y} & =0, \quad 0<x<1,-\infty<y<\infty, \\
u(0, y) & =g(y), \quad-\infty<y<\infty, \\
u_{x}(0, y) & =0, \quad-\infty<y<\infty,
\end{aligned}
$$

where we want to determine $u(x, y)$ for $0<x \leq 1$ from the data $g(y)$ with corresponding measured data function $g_{\delta}(y)$ [9-11].
In [9], the authors constructed a regularization method based on the Meyer wavelet, but the convergence rate of the method was not obtained. In [10, 11], a modification method and a Fourier method for this problem were given, respectively, and some error estimates with satisfactory convergence rates were also proved. However, some very important and difficult problems in the theoretical study, that is, the optimal error bound, are not discussed. The major object of this paper is to give the optimal error bounds in theory for problem (1) by employing a regularized theory based on spectral decomposition. Meanwhile we provide two optimal regularized methods, that is, the generalized Tikhonov regularization and the generalized singular value decomposition method, which realize the optimal error bound.

The motivation of this paper is inspired by Tautenhahn, in [12], where he discussed a Cauchy problem for the elliptic equation in a bounded domain and used the eigenvalues of the elliptic operator to express the exact solution of the problem. However, this method does not suit problem (1) in an unbounded strip region. Instead of using eigenvalues we employ the technique of Fourier transform.

Let $g(y)$ and $g_{\delta}(y)$ denote the exact and measured data, respectively, which satisfy

$$
\left\|g(\cdot)-g_{\delta}(\cdot)\right\| \leq \delta,
$$

where $\|\cdot\|$ denotes the $L^{2}$-norm and the noise level $\delta>0$ is determined by the accuracy of the instruments. We assume 
the $g(y)$ and other functions appearing in this paper with respect to variable $y$ belong to $L^{2}(\mathbb{R})$. Let

$$
\widehat{g}(\xi):=\frac{1}{\sqrt{2 \pi}} \int_{-\infty}^{\infty} g(y) e^{-i \xi y} d y
$$

denote the Fourier transform of function $g(y)$. We now analyze problem (1) in the frequency space. Taking Fourier transform for problem (1) with respect to the variable $y$, we get

$$
\begin{aligned}
\widehat{u}_{x x}(x, \xi)-\xi^{2} \widehat{u}(x, \xi) & =0, \quad 0<x<1, \quad \xi \in \mathbb{R}, \\
\widehat{u}(0, \xi) & =\widehat{g}(\xi), \quad \xi \in \mathbb{R}, \\
\widehat{u}(0, \xi) & =0, \quad \xi \in \mathbb{R} .
\end{aligned}
$$

The unique solution of (4) is [9-11]

$$
\widehat{u}(x, \xi)=\widehat{g}(\xi) \cosh (x \xi) .
$$

Due to Parseval formula, $\widehat{u}(x, \cdot) \in L^{2}(\mathbb{R})$, and therefore (5) implies that $g(\xi)$ must decay rapidly as $|\xi| \rightarrow \infty$. However, as the measurement data $\widehat{g}_{\delta}(\xi)$, we can not expect $\widehat{g}_{\delta}(\xi)$ has the same decay in high frequency components; that is, small errors in high frequency components can blow up and completely destroy the solution for $0<x \leq 1$, noting that the factor $\cosh (x \xi)$ in (5) increases exponentially as $|\xi| \rightarrow \infty$, so the problem (1) is severely ill-posed.

In order to obtain explicit stability estimate for problem (1), some "source condition" is needed. For this we introduce the Sobolev space $\left.H_{r}\right|_{r \in \mathbb{R}^{+}}$according to $H_{0}=L^{2}(\mathbb{R}), H_{r}=$ $\left\{v(y):\|v\|_{r}<\infty\right\}$, where

$$
\|v\|_{r}:=\left(\int_{-\infty}^{\infty}\left(1+\xi^{2}\right)^{r}|\widehat{v}(\xi)|^{2} d \xi\right)^{1 / 2}
$$

is the norm in $H_{r}$. We require the a priori smoothness condition for problem (1) concerning the unknown solution $u(x, y)$ according to

$$
\begin{aligned}
& u(x, y) \in M_{p, E}:=\left\{u(x, \cdot) \in L^{2}(\mathbb{R}) \mid\|u(1, \cdot)\|_{p}\right. \\
& \quad \leq E \text { for some } p \geq 0\} .
\end{aligned}
$$

This paper is organized as follows: In Section 2 we briefly recount some preliminary results, which are the basis of the discussion for other sections. In Section 3 we give the optimal error bounds between the exact solution and its regularized approximation, which depend on the noise level $\delta$ either in a Hölder continuous way or in a logarithmic continuous way. In Section 4 we discuss two concrete regularization methods, that is, the generalized Tikhonov regularization and the generalized singular value decomposition, where both regularization methods realize the optimal error bounds.

\section{Preliminary Result}

We consider arbitrary ill-posed inverse problem [12-17]

$$
A x=y,
$$

where $A \in \mathscr{L}(X, Y)$ is a linear injective bounded operator between infinite dimensional Hilbert spaces $X$ and $Y$ with nonclosed range $R(A)$ of $A$. We assume that $y^{\delta} \in Y$ are available noisy data with $\left\|y-y^{\delta}\right\| \leq \delta$. Any operator $R: Y \rightarrow$ $X$ can be considered as a special method for solving (8), and the approximate solution of (8) is given by $R y^{\delta}$. However, the convergent rate of $R y^{\delta}$ to $x$ can be arbitrarily slow without assuming additional quantitative a prior restrictions on the unknown solution $x$, which is typical for ill-posed problem.

Assume we want to solve (8), we have the a priori information that the exact solution satisfies a source condition; that is, $x$ belongs to the source set

$$
M_{\varphi, E}:=\left\{x \in E \mid x=\left[\varphi\left(A^{*} A\right)\right]^{1 / 2} v,\|v\| \leq E\right\},
$$

where the operator function $\varphi\left(A^{*} A\right)$ is well defined via spectral representation $[13,14]$ :

$$
\varphi\left(A^{*} A\right)=\int_{0}^{a} \varphi(\lambda) d E_{\lambda},
$$

where

$$
A^{*} A=\int_{0}^{a} \lambda d E_{\lambda}
$$

is the spectral decomposition of $A^{*} A,\left\{E_{\lambda}\right\}$ denotes the spectral family of the operator $A^{*} A$, and $a$ is a constant such that $\left\|A^{*} A\right\| \leq a$. In the case when $A: L^{2}(\mathbb{R}) \rightarrow L^{2}(\mathbb{R})$ is a multiplication operator, $A x(s)=\gamma(s) x(s)$, the operator function $\varphi\left(A^{*} A\right)$ attains the form

$$
\varphi\left(A^{*} A\right) x(s)=\varphi\left(|\gamma(s)|^{2}\right) x(s) .
$$

Let us assume that $R: Y \rightarrow X$ is an arbitrary mapping to approximately recover $x$ from $y^{\delta}$. Then the worst case error for $R$ under the a priori information $x \in M_{\phi, E}$ is $[16,17]$

$$
\begin{aligned}
& \Delta_{R}\left(\delta, M_{\varphi, E}, A\right) \\
& \quad:=\sup \left\{\left\|R y^{\delta}-x\right\| \mid x \in M_{\varphi, E},\left\|A x-y^{\delta}\right\| \leq \delta\right\} .
\end{aligned}
$$

This worst case error characterizes the maximal error of the method $R$ if the solution $x$ of problem (8) varies in the set $M_{\phi, E}$. The best possible worst case error (or the optimal bound) is defined as

$$
\Delta\left(\delta, M_{\varphi, E}, A\right):=\inf _{R} \Delta\left(\delta, M_{\varphi, E}, A\right),
$$

where the minimum is taken over all methods $R: Y \rightarrow X$. It can be shown ( $c f .[13,18])$ that the minimum in (14) is actually obtained and

$$
\Delta\left(\delta, M_{\varphi, E}, A\right)=\omega\left(\delta, M_{\varphi, E}, A\right)
$$


with the modulus of continuity defined by

$$
\omega\left(\delta, M_{\varphi, E}, A\right):=\sup \left\{\|x\| \mid x \in M_{\varphi, E},\|A x\| \leq \delta\right\} .
$$

In order to derive explicitly the optimal error bounds for the worst case error $\Delta_{R}\left(\delta, M_{\varphi, E}, A\right)$ defined in (13) and obtain optimality results for special regularization methods, we assume that the function $\varphi$ in (9) satisfies the following assumption.

Assumption 1 (see $[13,14])$. The function $\varphi(\lambda):(0, a] \rightarrow$ $(0, \infty)$ in $(14)$, where $a$ is a constant such that $\left\|A^{*} A\right\| \leq a$, is continuous and has the following properties:

(i) $\lim _{\lambda \rightarrow 0} \varphi(\lambda)=0$,

(ii) $\varphi(\lambda)$ is strong monotonically increasing on $(0, a]$,

(iii) $\rho(\lambda):=\lambda \varphi^{-1}(\lambda):(0, \varphi(a)] \rightarrow(0, a \varphi(a)]$ is strong convex.

Under Assumption 1, the next theorem gives us a formula for the optimal error bound.

Theorem 2 (see $[13,14])$. Let $M_{\varphi, E}$ be given by (14), let Assumption 1 be satisfied, and let $\delta^{2} / E^{2} \in \sigma\left(A^{*} A \varphi\left(A^{*} A\right)\right)$, where $\sigma\left(A^{*} A\right)$ denotes the spectrum of operator $A^{*} A$; then

$$
\omega(\delta, x)=E \sqrt{\rho^{-1}\left(\frac{\delta^{2}}{E^{2}}\right)} .
$$

In the following we consider two special methods: the method of generalized Tikhonov regularization and the method of generalized singular value decomposition.

For the method of generalized Tikhonov regularization, a regularized approximation $x_{\alpha}^{\delta}$ is determined by solving the minimization problem $[13,14,16,17]$

$$
\begin{aligned}
\min _{x \in X} J_{\alpha}(x), & \\
& J_{\alpha}(x)=\left\|A x-y^{\delta}\right\|^{2}+\alpha\left\|\left[\varphi\left(A^{*} A\right)\right]^{-1 / 2} x\right\|^{2},
\end{aligned}
$$

or equivalently, by solving the Euler equation

$$
\left(A^{*} A+\alpha\left[\varphi\left(A^{*} A\right)\right]^{-1}\right) x_{\alpha}^{\delta}=A^{*} y^{\delta},
$$

and the following statement holds.

Theorem 3 (see $[12,13])$. Let $M_{\varphi, E}$ be given by (14), let Assumption 1 be satisfied, let $\varphi(\lambda)^{\varphi}:(0, a] \rightarrow \mathbb{R}$ be two times differentiable, let $\rho(\lambda)$ be strong convex on $(0, \varphi(a)]$, and $\delta^{2} / E^{2} \leq a \varphi(a)$. If the regularization parameter $\alpha$ is chosen optimally by

$$
\begin{aligned}
& \alpha=\frac{\lambda_{0}}{\varphi^{-1}\left(\lambda_{0}\right) \varphi^{\prime}\left(\varphi^{-1}\left(\lambda_{0}\right)\right)}\left(\frac{\delta}{E}\right)^{2} \\
& \text { with } \lambda_{0}=\rho^{-1}\left(\frac{\delta^{2}}{E^{2}}\right),
\end{aligned}
$$

then, for the Tikhonov regularized solution $x_{\alpha}^{\delta}=R_{\alpha} y^{\delta}$ defined by (18) or (19), the optimal error estimate

$$
\Delta\left(\delta, R_{\alpha}\right) \leq E \sqrt{\rho^{-1}\left(\frac{\delta^{2}}{E^{2}}\right)}
$$

holds.

The regularized approximation $x_{\alpha}^{\delta}$ based on the method of generalized singular value decomposition is given by

$$
\begin{aligned}
x_{\alpha}^{\delta}=g_{\alpha}\left(A^{*} A\right) A^{*} y^{\delta} & \\
& \text { with } g_{\alpha}(\lambda)= \begin{cases}\frac{1}{\lambda}, & \text { for } \lambda \geq \alpha, \\
\frac{1}{\alpha}, & \text { for } \lambda \leq \alpha .\end{cases}
\end{aligned}
$$

For this method the following result holds $[13,14]$.

Theorem 4. Let $M_{\varphi, E}$ be given by (9), let Assumption 1 be satisfied, let $\varphi(\lambda):(0, a] \rightarrow \mathbb{R}$ be two times differentiable, let $\rho(\lambda)$ be strong convex on $(0, \varphi(a)]$, and $\delta^{2} / E^{2} \leq a \varphi(a)$. If the regularization parameter $\alpha$ is chosen optimally by

$$
\alpha=\frac{\varphi\left(\lambda_{0}\right)+\lambda_{0} \varphi^{\prime}\left(\lambda_{0}\right)}{\varphi^{\prime}\left(\lambda_{0}\right)} \quad \text { with } \lambda_{0} \varphi\left(\lambda_{0}\right)=\left(\frac{\delta}{E}\right)^{2},
$$

then for the regularized solution $x_{\alpha}^{\delta}=R_{\alpha} y^{\delta}$ defined by (22) the optimal error estimate (21) holds.

\section{Optimal Error Bounds for Problem (1)}

Let us formulate the problem (1) for identifying $u(x, y)$ from unperturbed data $u(0, y)$ as operator equation

$$
A(x) u(x, y)=u(0, y)
$$

with a linear operator $A(x) \in \mathscr{L}\left(L^{2}(\mathbb{R}), L^{2}(\mathbb{R})\right)$; then the equivalent operator equation of (24) in the Fourier domain is given by

$$
\widehat{A}(x) \widehat{u}(x, \xi)=\widehat{u}(0, \xi), \quad \widehat{A}(x)=\mathscr{F} A(x) \mathscr{F}^{-1},
$$

where $\mathscr{F}: L^{2}(\mathbb{R}) \rightarrow L^{2}(\mathbb{R})$ is the (unitary) Fourier operator that maps any $L^{2}(\mathbb{R})$ function $v(y)$ into its Fourier transform $\widehat{v}(\xi)$. From (5) and (25), we have

$$
\widehat{A}(x) \widehat{u}(x, \xi)=\frac{1}{\cosh (x \xi)} \widehat{u}(x, \xi),
$$

where $\widehat{A}(x): L^{2}(\mathbb{R}) \rightarrow L^{2}(\mathbb{R})$ is a multiplication operator. It is easy to know that the operator $\widehat{A}(x)$ is self-adjoint, so $\widehat{A}^{*}(x) \widehat{A}(x): L^{2}(\mathbb{R}) \rightarrow L^{2}(\mathbb{R})$ is given by

$$
\widehat{A}^{*}(x) \widehat{A}(x)=\frac{1}{\cosh ^{2}(x \xi)} .
$$


Due to Parseval formula, $u(x, y) \in M_{p, E}$ is equivalent to $\widehat{u}(x, \xi) \in \widehat{M}_{p, E}$, where

$$
\begin{aligned}
& \widehat{u}(x, \xi) \in \widehat{M}_{p, E}:=\left\{\widehat{u}(x, \cdot) \in L^{2}(\mathbb{R}) \mid\|\widehat{u}(1, \cdot)\|_{p}\right. \\
& \quad \leq E \text { for some } p \geq 0\}
\end{aligned}
$$

with

$$
\|\widehat{u}(1, \cdot)\|_{p}:=\left(\int_{-\infty}^{+\infty}\left(1+\xi^{2}\right)|\widehat{u}(1, \xi)|^{2} d \xi\right)^{1 / 2} .
$$

Note that the source condition (9) for problem (1) can be written as

$$
\begin{aligned}
& M_{\varphi, E}=\{u(x, \cdot) \\
& \left.\quad \in L^{2}(\mathbb{R}) \mid\left\|\left[\varphi\left(A^{*}(x) A(x)\right)\right]^{-1 / 2} u(x, \cdot)\right\| \leq E\right\},
\end{aligned}
$$

and then its equivalent form in Fourier frequency space is given by

$$
\begin{aligned}
& \widehat{M}_{\varphi, E}=\{\widehat{u}(x, \cdot) \\
& \left.\quad \in L^{2}(\mathbb{R}) \mid\left\|\left[\varphi\left(\widehat{A}^{*}(x) \widehat{A}(x)\right)\right]^{-1 / 2} \widehat{u}(x, \cdot)\right\| \leq E\right\} .
\end{aligned}
$$

Due to the equivalence of conditions (30) and (7), we know the conditions (28) and (31) are equivalent and we have the following result.

Proposition 5. For operator equation (25), the set $M_{p, E}$ given by (7) is equivalent to the general source set $M_{\varphi, E}$ given by (30) provided $\varphi=\varphi(\lambda)$ is given (in parameter representation) by

$$
\begin{aligned}
& \lambda(\xi)=\frac{1}{\cosh ^{2}(x \xi)}, \\
& \varphi(\xi)=\left(1+\xi^{2}\right)^{-p} \frac{\cosh ^{2}(x \xi)}{\cosh ^{2}(\xi)},
\end{aligned}
$$

$$
\xi \in \mathbb{R}, 0<x \leq 1 .
$$

Proof. From (5) we have

$$
\widehat{u}(1, \xi)=\widehat{g}(\xi) \cosh \xi,
$$

which gives

$$
\widehat{u}(1, \xi)=\frac{\cosh \xi}{\cosh (x \xi)} \widehat{u}(x, \xi),
$$

and then the inequality $\|u(1, y)\|_{p} \leq E$ is equivalent to

$$
\begin{aligned}
\left\|\left(1+\xi^{2}\right)^{p / 2} \widehat{u}(1, \xi)\right\| \\
\quad=\left\|\left(1+\xi^{2}\right)^{p / 2} \frac{\cosh \xi}{\cosh (x \xi)} \widehat{u}(x, \xi)\right\| \leq E .
\end{aligned}
$$

Note that the set $\widehat{M}_{p, E}$ given by (28) is equivalent to the set $\widehat{M}_{\varphi, E}$ given in (31), we know (35) is equivalent to

$$
\left\|\left[\varphi\left(\widehat{A}^{*}(x) \widehat{A}(x)\right)\right]^{-1 / 2} \widehat{u}(x, \xi)\right\| \leq E .
$$

So, we obtain that the operator function $\varphi=\varphi(\lambda)$ in (30) has the representation

$$
\varphi\left(\widehat{A}^{*}(x) \widehat{A}(x)\right)=\left(1+\xi^{2}\right)^{-p} \frac{\cosh ^{2}(x \xi)}{\cosh ^{2} \xi} .
$$

Together with (27), $\varphi$ is given by (32) in its parameter representation. The proof is complete.

The function $\varphi=\varphi(\lambda)$ defined by (32) possesses the following properties.

Proposition 6. The function $\varphi=\varphi(\lambda)$ defined by (32) is continuous and satisfies the properties:

(i) $\lim _{\lambda \rightarrow 0} \varphi(\lambda)=0$.

(ii) $\varphi(\lambda)$ is strong monotonically increasing.

(iii) $\rho(\lambda)=\lambda \varphi^{-1}(\lambda)$ is strong monotonically increasing and possesses the parameter representation:

$$
\begin{aligned}
& \lambda(\xi)=\left(1+\xi^{2}\right)^{-p} \frac{\cosh ^{2}(x \xi)}{\cosh ^{2} \xi}, \\
& \rho(\xi)=\left(1+\xi^{2}\right)^{-p} \frac{1}{\cosh ^{2} \xi},
\end{aligned}
$$

$$
\xi \in \mathbb{R}, 0<x \leq 1 .
$$

(iv) $\rho^{-1}(\lambda)$ is strong monotonically increasing and possesses the parameter representation:

$$
\begin{aligned}
\lambda(\xi) & =\left(1+\xi^{2}\right)^{-p} \frac{1}{\cosh ^{2} \xi}, \\
\rho^{-1}(\xi) & =(1+\xi)^{-p} \frac{\cosh ^{2}(x \xi)}{\cosh ^{2} \xi},
\end{aligned}
$$

$$
\xi \in \mathbb{R}, 0<x \leq 1
$$

(v) For the inverse function $\rho^{-1}(\lambda)$ of $\rho(\lambda)$ the following holds:

$$
\rho^{-1}(\lambda)=\left(\frac{\lambda}{4}\right)^{1-x}\left[\ln \frac{1}{\sqrt{\lambda}}\right]^{-2 p x}(1+o(1)),
$$

$$
\text { for } \lambda \longrightarrow 0
$$

for any fixed $x \in(0,1]$.

(vi) The function $\rho$ defined by (38) is strong convex for $p=$ $0, x \in(0,1)$ and $p>0, x \in(0,1]$.

Proof. The continuity of function $\varphi(\lambda)$ is obvious.

(i) From (32) we have

$$
\dot{\lambda}(\xi)=-\frac{2 x \sinh (x \xi)}{\cosh ^{3}(x \xi)}=-2 \lambda(\xi) x \tanh (x \xi) .
$$


It is easy to know that $\dot{\lambda}(\xi) \leq 0$ for $\xi \geq 0$; that is, the function $\lambda(\xi)$ is decreasing and $\lim _{\xi \rightarrow+\infty} \lambda(\xi)=0$, so,

$$
\lim _{\lambda \rightarrow 0} \varphi(\lambda)=\lim _{\xi \rightarrow+\infty} \varphi(\xi)=0 .
$$

Moreover, when $\xi \leq 0, \dot{\lambda}(\xi) \geq 0$, we know $\lambda(\xi)$ is increasing and $\lim _{\xi \rightarrow-\infty} \lambda(\xi)=0$,

$$
\lim _{\lambda \rightarrow 0} \varphi(\lambda)=\lim _{\xi \rightarrow-\infty} \varphi(\xi)=0 .
$$

From (42) and (43), we have $\lim _{\lambda \rightarrow 0} \varphi(\lambda)=0$.

(ii) From (32), we know

$$
\dot{\varphi}(\xi)=\varphi(\xi)\left(-\frac{2 p \xi}{1+\xi^{2}}+2 x \tanh (x \xi)-2 \tanh \xi\right) .
$$

Together with (41), we have

$$
\begin{aligned}
\varphi^{\prime}(\lambda) & =\frac{\dot{\varphi}(\xi)}{\dot{\lambda}(\xi)} \\
= & \frac{\varphi(\xi)}{\lambda(\xi)} \frac{2 p \xi /\left(1+\xi^{2}\right)+2 \tanh \xi-2 x \tanh (x \xi)}{2 x \tanh (x \xi)} .
\end{aligned}
$$

It is easy to know that $\varphi(\xi)$ and $\lambda(\xi)$ are all even functions about variable $\xi$; therefore $\varphi^{\prime}(\lambda)$ is also so. Here it is only to consider the case $\xi \geq 0$. Note that the function $f(t):=$ $\tanh t$ is strong monotonically increasing; together with (32) we know $\varphi^{\prime}(\xi)>0$ for $\xi>0$. Moreover, a straightforward computation gives

$$
\begin{aligned}
\lim _{\xi \rightarrow 0^{+}} & \frac{p \xi /\left(1+\xi^{2}\right)+\tanh \xi-x \tanh (x \xi)}{x \tanh (x \xi)} \\
& =\frac{p-x^{2}+1}{x^{2}} .
\end{aligned}
$$

So, we can easily see $\varphi^{\prime}(\lambda)>0$ for $p=0, x \in(0,1)$ and $p>0$, $x \in(0,1]$; that is, when $p=0, x \in(0,1)$ and $p>0, x \in(0,1]$, $\varphi(\lambda)$ is strong monotonically increasing.

(iii) From (ii) we know that $\varphi^{-1}(\lambda)$ is strong monotonically increasing for $p=0, x \in(0,1)$ and $p>0, x \in(0,1]$. Therefore, when $p=0, x \in(0,1)$ and $p>0, x \in(0,1], \rho(\lambda)=$ $\lambda \varphi^{-1}(\lambda)$ is also strong monotonically increasing. From (32), it is easy to know that $\varphi^{-1}(\lambda)$ has the parameter representation

$$
\begin{aligned}
\lambda(\xi) & =\left(1+\xi^{2}\right)^{-p} \frac{\cosh ^{2}(x \xi)}{\cosh ^{2} \xi}, \\
\varphi^{-1}(\xi) & =\frac{1}{\cosh ^{2}(x \xi)},
\end{aligned}
$$

$$
\xi \in \mathbb{R}, 0<x \leq 1 \text {. }
$$

So, the parameter expression (38) of function $\rho(\lambda)$ holds.

(iv) According to (iii), $\rho^{-1}(\lambda)$ is also strong monotonically increasing and its parameter representation (39) can be obtained from (38) immediately. (v) From (39) we have

$$
\begin{aligned}
\ln \frac{1}{\lambda} & =p \ln \left(1+\xi^{2}\right)+2 \xi+\ln \frac{1+e^{-4 \xi}+2 e^{-2 \xi}}{2} \\
& =2 \xi(1+0(1)) \quad \text { for } \xi \longrightarrow \infty,
\end{aligned}
$$

and consider

$$
e^{2 \xi}=\frac{4}{\lambda}\left(1+\xi^{2}\right)^{-p}(1+o(1))
$$

for $\xi \longrightarrow \infty$, that is, $\lambda \longrightarrow 0$.

It is easy to see that

$$
\xi=\ln \frac{1}{\sqrt{\lambda}}(1+o(1)), \quad \text { for } \lambda \longrightarrow 0 .
$$

Inserting (49) and (50) in (39), we have

$$
\begin{aligned}
\rho^{-1}(\xi) & =\left(1+\xi^{2}\right)^{-p} \frac{\cosh ^{2}(x \xi)}{\cosh ^{2} \xi} \\
& =\lambda(\xi) \frac{e^{2 x \xi}+e^{-2 x \xi}+2}{4} \\
& =\frac{\lambda}{4} e^{2 x \xi}(1+o(1)) \\
& =\left(\frac{\lambda}{4}\right)^{1-x}\left(1+\xi^{2}\right)^{-p x}(1+o(1)) \\
& \text { for } \lambda \longrightarrow 0, \text { that is, for } \xi \longrightarrow \infty \\
& \text { for } \lambda \longrightarrow 0, \text { that is, for } \xi \longrightarrow \infty \\
& \left(\frac{\lambda}{4}\right)^{1-x}\left(\ln \frac{1}{\sqrt{\lambda}}\right)^{-2 p x}(1+o(1))
\end{aligned}
$$$$
\text { for } \lambda \longrightarrow 0 \text {. }
$$

Indeed, if we denote

$$
F(\lambda):=\rho^{-1}(\lambda)\left(\frac{\lambda}{4}\right)^{x-1}\left(\ln \frac{1}{\sqrt{\lambda}}\right)^{2 p x}
$$

it is easy to prove that

$$
\begin{aligned}
\lim _{\lambda \rightarrow 0} F(\lambda) & =\lim _{\xi \rightarrow \infty} \rho^{-1}(\xi)\left(\frac{\lambda(\xi)}{4}\right)^{x-1}\left(\ln \frac{1}{\sqrt{\lambda(\xi)}}\right)^{2 p x} \\
& =1 .
\end{aligned}
$$

So, the representation (40) holds.

(vi) We know that the function $\rho(\lambda)$ is strong convex if and only if $\rho^{\prime \prime}(\lambda)>0$. Denoting $\lambda(\xi)=\rho(\xi) r(\xi)$ with $r(\xi)=$ $\cosh ^{2}(x \xi)$, from (38) we have

$$
\rho^{\prime \prime}(\lambda)=\frac{\ddot{\rho} \dot{\lambda}-\dot{\rho} \ddot{\lambda}}{\dot{\lambda}^{3}}=\frac{\ddot{\rho} \rho \dot{r}-2 \dot{\rho}^{2} \dot{r}-\rho \dot{\rho} \ddot{r}}{(\dot{\rho} r+\rho \dot{r})^{3}},
$$


where

$$
\begin{aligned}
\dot{\rho} & =\dot{\rho}(\xi)=-2 \rho(\xi)\left[\frac{p \xi}{1+\xi^{2}}+\tanh \xi\right], \\
\ddot{\rho}= & \ddot{\rho}(\xi)=-2 \rho(\xi)\left\{-2\left[\frac{p \xi}{1+\xi^{2}}+\tanh \xi\right]^{2}\right. \\
& \left.+\frac{p\left(1-\xi^{2}\right)}{\left(1+\xi^{2}\right)^{2}}+\frac{1}{\cosh ^{2} \xi}\right\}, \\
\dot{r}= & \dot{r}(\xi)=x \sinh (x \xi), \\
\ddot{r}= & \ddot{r}(\xi)=2 x^{2} \cosh (2 x \xi) .
\end{aligned}
$$

It is easy to know that $\rho^{\prime \prime}(\lambda)$ is even function about variable $\xi$; therefore we only need to consider the case $\xi \geq 0$.

1st Case $(\xi>0)$. In this case, $\lambda^{\prime}(\xi)<0$. Then $\rho^{\prime \prime}(\lambda)>0$ is equivalent to $\ddot{\rho} \dot{\lambda}<\dot{\rho} \ddot{\lambda}$, that is,

$$
\rho \ddot{\rho}-2 \dot{\rho}^{2}<\rho \dot{\rho} \frac{\ddot{r}}{\dot{r}} .
$$

A straightforward computation shows that (56) is equivalent to

$$
\begin{aligned}
& \left(\frac{\xi}{1+\xi^{2}}\right)^{2} p^{2}+p\left\{\frac{2 \xi}{1+\xi^{2}} \tanh \xi+\frac{1-\xi^{2}}{2\left(1+\xi^{2}\right)^{2}}\right. \\
& \left.\quad-\frac{x \xi}{1+\xi^{2}} \operatorname{coth}(2 x \xi)\right\}+\tanh ^{2} \xi+\frac{1}{2 \cosh ^{2} \xi}-x \\
& \quad \cdot \tanh \xi \operatorname{coth}(2 x \xi)>0 .
\end{aligned}
$$

From (57) we have

$$
\begin{aligned}
& \tanh ^{2} \xi+\frac{1}{2 \cosh ^{2} \xi}-x \tanh \xi \operatorname{coth}(2 x \xi) \\
& =\frac{2 \sinh ^{2} \xi+1}{2 \cosh ^{2} \xi}-\frac{2 \sinh ^{2}(x \xi)+1}{2 \cosh ^{2}(x \xi)} \cdot \frac{x \tanh \xi}{\tanh (x \xi)} \\
& =\frac{2 \sinh ^{2} \xi+1}{2 \cosh ^{2} \xi}-\frac{2 \sinh ^{2}(x \xi)+1}{2 \cosh ^{2}(x \xi)} \cdot \frac{\tanh \xi / \xi}{\tanh (x \xi) / x \xi}
\end{aligned}
$$

Note that the function $f_{1}(t):=\left(2 \sinh ^{2} t+1\right) / 2 \cosh ^{2} t$ is monotonically increasing and $f_{2}(t):=\tanh t / t$ is monotonically decreasing, and we obtain that

$$
\tanh ^{2} \xi+\frac{1}{2 \cosh ^{2} \xi}-x \tanh \xi \operatorname{coth}(2 x \xi)>0
$$

for all $x \in(0,1]$. So, when $\xi>0$, the function $\rho(\lambda)$ is strong convex if

$$
\begin{aligned}
& \left(\frac{\xi}{1+\xi^{2}}\right)^{2} p^{2}+p\left\{\frac{2 \xi}{1+\xi^{2}} \tanh \xi+\frac{1-\xi^{2}}{2\left(1+\xi^{2}\right)^{2}}\right. \\
& \left.-\frac{x \xi}{1+\xi^{2}} \operatorname{coth}(2 x \xi)\right\}>0,
\end{aligned}
$$

that is,

$$
\begin{aligned}
p+\frac{2\left(1+\xi^{2}\right)}{\xi} \tanh \xi+\frac{1-\xi^{2}}{2 \xi^{2}} & \\
- & \frac{x\left(1+\xi^{2}\right)}{\xi} \operatorname{coth}(2 x \xi)>0 .
\end{aligned}
$$

Note that the function $f_{3}(x):=\left(x\left(1+\xi^{2}\right) / \xi\right) \operatorname{coth}(2 x \xi)$ is increasing about $x$ for $\xi>0$; by numerical computation we know

$$
\begin{aligned}
& \frac{2\left(1+\xi^{2}\right)}{\xi} \tanh \xi+\frac{1-\xi^{2}}{2 \xi^{2}}-\frac{x\left(1+\xi^{2}\right)}{\xi} \operatorname{coth}(2 x \xi) \\
& \quad \geq \frac{1}{3} .
\end{aligned}
$$

So, when $p \geq 0,(60)$ holds naturally. With (57) and (57)' together, we know that $\rho(\lambda)$ is strong convex for $p \geq 0, \xi>0$ and $x \in(0,1]$.

2nd Case $(\xi=0)$. An elementary calculation shows

$$
\begin{aligned}
\lim _{\xi \rightarrow 0^{+}} \frac{\ddot{\rho} \rho \dot{r}-2 \dot{\rho}^{2} \dot{r}-\rho \dot{\rho} \ddot{r}}{(\dot{\rho} r+\rho \dot{r})^{3}} \\
\quad=\frac{16 p\left[-3(p+1)+2 x^{4}\right]-32\left(x^{2}-x^{4}\right)}{6\left[-2(p+1)+2 x^{2}\right]}>0
\end{aligned}
$$

for all $p \geq 0$ and $x \in(0,1)$. From above, (vi) is proved.

The proof is finished.

Now we formulate our main result of this section concerning the best possible worst case error $\omega(\delta, x)$ defined by (17) for identifying solution $u(x, y)$ of problem (1) from noisy data $u^{\delta}(0, y)$ under the condition (2) and $u(x, y) \in M_{p, E}$ where the set $M_{p, E}$ is given by (7). We denote

$$
\omega(\delta, x):=\Delta\left(\delta, M_{p, E}, A(x)\right)
$$

with $\Delta\left(\delta, M_{p, E}, A(x)\right)$ given as $\Delta\left(\delta, M_{\varphi, E}, A(x)\right)$ in (14). Due to Parseval identity and the equivalence of $\widehat{M}_{p, E}$ which are given by (28) and (31), respectively, we have

$$
\begin{aligned}
\omega(\delta, x) & =\Delta\left(\delta, \widehat{M}_{p, E}, \widehat{A}(x)\right)=\Delta\left(\delta, \widehat{M}_{\varphi, E}, \widehat{A}(x)\right) \\
& =\omega\left(\delta, \widehat{M}_{p, E}, \widehat{A}(x)\right) .
\end{aligned}
$$

Applying Theorem 2 and Proposition 6 we have the following result.

Theorem 7. Let $\delta^{2} / E^{2} \leq 1$, and then consider the following.

(i) In case $p=0$ and $0<x<1$, the following holds:

$$
\omega(\delta, x)=E^{x}\left(\frac{\delta}{2}\right)^{1-x}(1+o(1)), \quad \text { for } \delta \longrightarrow 0
$$

(Hölder stability). 
(ii) In case $p>0$ and $x=1$, the following holds:

$$
\omega(\delta, 1)=E\left[\ln \frac{E}{\delta}\right]^{-p}(1+o(1)), \quad \text { for } \delta \longrightarrow 0
$$

(logarithmic stability).

(iii) In case $p>0$ and $0<x<1$, the following holds:

$$
\omega(\delta, x)=E^{x}\left(\frac{\delta}{2}\right)^{1-x}\left[\ln \frac{E}{\delta}\right]^{-p x}(1+o(1))
$$

$$
\text { for } \delta \longrightarrow 0 \text {. }
$$

Proof. From (32), we have

$$
\widehat{A}(x)^{*} \widehat{A}(x) \varphi\left(\widehat{A}(x)^{*} \widehat{A}(x)\right)(\xi)=\frac{\left(1+\xi^{2}\right)^{-p}}{\cosh ^{2} \xi} .
$$

It is easy to know the function $f_{4}(\xi):=\left(1+\xi^{2}\right)^{-p} / \cosh ^{2} \xi$ takes maximum at $\xi=0$, and $\lim _{\xi \rightarrow \infty} f_{4}(\xi)=0$, so $\sigma\left(\widehat{A}(x)^{*} \widehat{A}(x) \varphi\left(\widehat{A}(x)^{*} \widehat{A}(x)\right)\right) \subseteq(0,1]$. Due to Theorem 2 and (40) given in Proposition 6, we know if $\delta^{2} / E^{2} \leq 1$.

(i) For $p=0$ and $0<x<1$,

$$
\begin{aligned}
\omega(\delta, x) & =E \cdot\left\{\left.\left(\frac{\lambda}{4}\right)^{(1-x)}\right|_{\lambda=\delta^{2} / E^{2}}\right\}^{1 / 2}(1+o(1)) \\
& =E^{x}\left(\frac{\delta}{2}\right)^{1-x}(1+o(1)), \quad \text { for } \delta \longrightarrow 0
\end{aligned}
$$

(Hölder stability). (ii) In case $p>0$ and $x=1$,

$$
\begin{aligned}
\omega(\delta, 1) & =E \cdot\left\{\left.\left(\ln \frac{1}{\lambda}\right)^{-2 p}\right|_{\lambda=\delta^{2} / E^{2}}\right\}^{1 / 2}(1+o(1)) \\
& =E\left[\ln \frac{E}{\delta}\right]^{-p}(1+o(1)), \quad \text { for } \delta \longrightarrow 0
\end{aligned}
$$

(logarithmic stability).

(iii) In case $p>0$ and $0<x<1$,

$\omega(\delta, x)$

$$
\begin{aligned}
& =E \cdot\left\{\left.\left(\frac{\lambda}{4}\right)^{1-x}\left[\ln \frac{1}{\lambda}\right]^{-2 p x}\right|_{\lambda=\delta^{2} / E^{2}}\right\}^{1 / 2}(1+o(1)) \\
& =E^{x}\left(\frac{\delta}{2}\right)^{1-x}\left[\ln \frac{E}{\delta}\right]^{-p x}(1+o(1)),
\end{aligned}
$$

for $\delta \longrightarrow 0$

The proof is complete.

\section{Optimal Regularization Methods}

In this section we consider two special regularization methods, apply them to problem (1), and show how to choose the regularization parameter such that it guarantees the optimal error bounds given by (65)-(67).

The method of generalized Tikhonov regularization (18) consists in the determination of a regularized approximation $u_{\alpha}^{\delta}=u_{\alpha}^{\delta}(x, y)$ by solving the minimization problem:

$$
\min _{\widehat{u} \in \widehat{M}_{\varphi, E}} J_{\alpha}(u), \quad J_{\alpha}(\widehat{u})=\left\|\frac{1}{\cosh (x \xi)} \widehat{u}(x, \xi)-\widehat{u}^{\delta}(0, \xi)\right\|^{2}+\alpha\left\|\left(1+\xi^{2}\right)^{p / 2} \frac{\cosh (\xi)}{\cosh (x \xi)} \widehat{u}(x, \xi)\right\|^{2},
$$

or, equivalently, it is the solution of Euler equation:

$$
\begin{aligned}
& \left(\frac{1}{\cosh ^{2}(x \xi)}+\alpha\left(1+\xi^{2}\right)^{p} \frac{\cosh ^{2} \xi}{\cosh ^{2}(x \xi)}\right) \widehat{u}_{\alpha}^{\delta}(x, \xi) \\
& =\frac{1}{\cosh (x \xi)} \widehat{u}^{\delta}(0, \xi) .
\end{aligned}
$$

Applying Theorem 3 and Proposition 5 to problem (1), we obtain the following result.

Theorem 8. Consider the operator equation (24) and assume its unknown solution $u(x, y) \in M_{p, E}$ given by (7). If $\delta^{2} / E^{2} \leq 1$ holds, then the method of generalized Tikhonov regularization (72) or (73) is optimal on $M_{p, E}$ provided that the regularization parameter $\alpha$ is chosen optimally by

$$
\alpha_{0}=\frac{x \tanh \left(x \xi_{0}\right)}{p \xi_{0} /\left(1+\xi_{0}^{2}\right)+\tanh \xi_{0}-x \tanh \left(x \xi_{0}\right)}\left(\frac{\delta}{E}\right)^{2},
$$

where $\xi_{0}$ is the unique solution of the equation

$$
\frac{\left(1+\xi^{2}\right)^{-p}}{\cosh ^{2} \xi}=\frac{\delta^{2}}{E^{2}}
$$

(i) In the case $x=1$ and $p>0$ the following holds:

$$
\alpha_{0}=\frac{1}{p}\left[\ln \frac{E}{\delta}\right]\left(\frac{\delta}{2}\right)^{2}(1+o(1)), \quad \text { for } \delta \longrightarrow 0
$$

(ii) In the case $p \geq 0$ and $0<x<1$, the following holds:

$$
\alpha_{0}=\frac{x}{1-x}\left(\frac{\delta}{2}\right)^{2}(1+o(1)), \quad \text { for } \delta \longrightarrow 0
$$

Furthermore, the optimal error estimate $\left\|u_{\alpha}^{\delta}(x, y)-u(x, y)\right\| \leq$ $\omega(\delta, x)$ holds, where $\omega(\delta, x)$ is given by (65)-(67), respectively. 
Proof. From Theorem 3, it follows that the optimal regularization parameter $\alpha$ is given by (20) with $\varphi(\lambda)$ given by (32), which is equivalent to

$$
\begin{aligned}
\alpha_{0} & =\frac{\varphi\left(\lambda_{0}\right)}{\lambda_{0} \varphi^{\prime}\left(\lambda_{0}\right)}\left(\frac{\delta}{E}\right)^{2}=\frac{\varphi\left(\xi_{0}\right) \dot{\lambda}\left(\xi_{0}\right)}{\lambda\left(\xi_{0}\right) \dot{\varphi}\left(\xi_{0}\right)}\left(\frac{\delta}{E}\right)^{2} \\
& =\frac{x \tanh \left(x \xi_{0}\right)}{p \xi_{0} /\left(1+\xi_{0}^{2}\right)+\tanh \xi_{0}-x \tanh \left(x \xi_{0}\right)}\left(\frac{\delta}{E}\right)^{2},
\end{aligned}
$$

with

$$
\lambda_{0}=\rho^{-1}\left(\frac{\delta^{2}}{E^{2}}\right)=\frac{\left(1+\xi^{2}\right)^{-p}}{\cosh ^{2} \xi} ;
$$

that is, $\xi_{0}$ is the unique solution of (75).

(i) In the case $x=1$ and $p>0$, note that $\xi_{0} \rightarrow \infty$ for $\delta \rightarrow 0$, and tanh $x \rightarrow 1$ for $x \rightarrow \infty$, and from (78) and (50), we have

$$
\begin{aligned}
\alpha_{0} & =\frac{x \tanh \xi_{0}}{p \xi_{0} /\left(1+\xi_{0}^{2}\right)}\left(\frac{\delta}{E}\right)^{2}=\frac{\xi_{0}}{p}\left(\frac{\delta}{E}\right)^{2}(1+o(1)) \\
& =\frac{1}{p}\left[\ln \frac{E}{\delta}\right](1+o(1)) \quad \text { for } \delta \longrightarrow 0 .
\end{aligned}
$$

(ii) In the case $p \geq 0$ and $0<x<1$, the following holds:

$$
\begin{aligned}
\alpha_{0} & =\frac{x \tanh \left(x \xi_{0}\right)}{p \xi_{0} /\left(1+\xi_{0}^{2}\right)+\tanh \xi_{0}-x \tanh \left(x \xi_{0}\right)}\left(\frac{\delta}{E}\right)^{2} \\
& =\frac{x}{1-x}\left(\frac{\delta}{E}\right)^{2}(1+o(1)), \quad \text { for } \delta \longrightarrow 0 .
\end{aligned}
$$

The proof is finished.

Now we consider the method of generalized singular value decomposition. Due to (22), the regularized approximation $u_{\alpha}^{\delta}(x, y)$ is given by

$$
\begin{aligned}
& \widehat{u}_{\alpha}^{\delta}(x, \xi) \\
& = \begin{cases}\left(\widehat{A}^{*}(x) \widehat{A}(x)\right)^{-1} \widehat{A}^{*}(x) \widehat{u}^{\delta}(0, \xi), & \left\|\widehat{A}^{*}(x) \widehat{A}(x)\right\| \geq \alpha, \\
\frac{1}{\alpha} \widehat{A}^{*}(x) \widehat{u}^{\delta}(0, \xi), & \left\|\widehat{A}^{*}(x) \widehat{A}(x)\right\| \leq \alpha,\end{cases} \\
& = \begin{cases}\cosh (x \xi) \widehat{u}^{\delta}(0, \xi), & \frac{1}{\cosh ^{2}(x \xi)} \geq \alpha, \\
\frac{1}{\alpha} \frac{1}{\cosh (x \xi)} \widehat{u}^{\delta}(0, \xi), & \frac{1}{\cosh ^{2}(x \xi)} \leq \alpha .\end{cases}
\end{aligned}
$$
following.

Theorem 9. Consider the operator equation (24) and assume its unknown solution $u(x, y) \in M_{p, E}$ given by (7). If $\delta^{2} / E^{2} \leq 1$ holds, then the method of generalized singular value decomposition (82) is optimal on $M_{p, E}$ provided that the regularization parameter $\alpha$ is chosen optimally by

$$
\alpha_{0}=\frac{p \xi_{0} /\left(1+\xi_{0}^{2}\right) \tanh \xi_{0}}{\cosh ^{2}\left(x \xi_{0}\right)\left(\tanh \xi_{0}-x \tanh \left(x \xi_{0}\right)\right)},
$$

where $\xi_{0}$ is the unique solution of (75). (i) In the case $x=1$ and $p>0$, the following holds:

$$
\alpha_{0}=\frac{1}{p}\left[\ln \frac{E}{\delta}\right]^{2 p+1}\left(\frac{\delta}{E}\right)^{2}(1+o(1)) \quad \text { for } \delta \longrightarrow 0 .
$$

(ii) In the case $0<x<1, p \geq 0$, the following holds:

$$
\alpha_{0}=\frac{4^{1-x}}{1-x}\left[\ln \frac{E}{\delta}\right]^{2 p x}\left(\frac{\delta}{E}\right)^{2 x}(1+o(1)),
$$

for $\delta \longrightarrow 0$.

Furthermore, the optimal error estimate $\left\|u_{\alpha}^{\delta}(x, y)-u(x, y)\right\| \leq$ $\omega(\delta, x)$ holds, where $\omega(\delta, x)$ is given by (65)-(67), respectively.

Proof. From Theorem 4, we know that the optimal regularization parameter $\alpha$ is given by (23) with $\varphi(\lambda)$ given by (32), which is equivalent to

$$
\begin{aligned}
& \alpha_{0}=\frac{\varphi\left(\lambda_{0}\right)+\lambda_{0} \varphi^{\prime}\left(\lambda_{0}\right)}{\varphi^{\prime}\left(\lambda_{0}\right)} \\
& =\frac{\varphi\left(\xi_{0}\right)+\lambda\left(\xi_{0}\right) \dot{\varphi}\left(\xi_{0}\right) / \dot{\lambda}\left(\xi_{0}\right)}{\dot{\varphi}\left(\xi_{0}\right) / \dot{\lambda}\left(\xi_{0}\right)} \\
& =\frac{\dot{\lambda}\left(\xi_{0}\right) \varphi\left(\xi_{0}\right)+\lambda\left(\xi_{0}\right) \dot{\varphi}\left(\xi_{0}\right)}{\dot{\varphi}\left(\xi_{0}\right)} \\
& =\frac{p \xi_{0} /\left(1+\xi_{0}^{2}\right)+\tanh \xi_{0}}{\cosh ^{2}\left(x \xi_{0}\right)\left(p \xi_{0} /\left(1+\xi_{0}^{2}\right)+\tanh \xi_{0}-x \tanh \left(x \xi_{0}\right)\right)}
\end{aligned}
$$

where $\xi_{0}$ is the unique solution of (75).

(i) In the case $x=1$ and $p>0$, note that $\xi_{0} \rightarrow \infty$ for $\delta \rightarrow 0$, and tanh $x \rightarrow 1$ for $x \rightarrow \infty$, and from (83) and (75), we have

$$
\begin{aligned}
\alpha_{0} & =\frac{p \xi_{0} /\left(1+\xi_{0}^{2}\right)+\tanh \xi_{0}}{\cosh ^{2}\left(x \xi_{0}\right)\left(p \xi_{0} /\left(1+\xi_{0}^{2}\right)\right)} \\
& =\frac{\left(p \xi_{0} /\left(1+\xi_{0}^{2}\right)+1\right)\left(1+\xi_{0}\right)^{p}}{=p \xi_{0} /\left(1+\xi_{0}^{2}\right)}\left(\frac{\delta}{E}\right)^{2}(1+o(1)) \\
& \quad \text { for } \xi_{0} \longrightarrow \infty, \text { that is, } \delta \longrightarrow 0 \\
& =\frac{1}{p} \xi_{0}^{2 p+1}\left(\frac{\delta}{E}\right)^{2}(1+o(1)) \\
& =\frac{1}{p}\left[\ln \frac{E}{\delta}\right]^{2 p+1}\left(\frac{\delta}{E}\right)^{2}(1+o(1)) \quad \text { for } \delta \longrightarrow 0 .
\end{aligned}
$$


(ii) In the case $p \geq 0$ and $0<x<1$, from (75) and (83), we have

$$
\begin{aligned}
\alpha_{0} & =\frac{\tanh \xi_{0}}{\cosh ^{2}\left(x \xi_{0}\right)\left(\tanh \xi_{0}-x \tanh \left(x \xi_{0}\right)\right)} \\
& =4 e^{-2 x \xi_{0}} \frac{1}{1-x}(1+o(1)), \\
& \text { for } \xi_{0} \longrightarrow \infty, \text { that is, } \delta \longrightarrow 0 \\
& =\frac{4^{1-x}}{1-x}\left(1+\xi_{0}^{2}\right)^{p x}\left(\frac{\delta}{E}\right)^{2 x}, \\
& =\frac{4^{1-x}}{1-x}\left[\ln \frac{E}{\delta}\right]^{2 p x}\left(\frac{\delta}{E}\right)^{2 x}(1+o(1)), \quad \text { for } \xi_{0} \longrightarrow \infty, \text { that is, } \delta \longrightarrow 0 \\
& \text { for } \delta \longrightarrow 0 .
\end{aligned}
$$

Furthermore, the optimal error estimate $\| u_{\alpha_{0}}^{\delta}(x, y)$ $u(x, y) \| \leq \omega(\delta, x)$ holds, where $\omega(\delta, x)$ is given by $(65)-(67)$, respectively. This completes the proof.

\section{Competing Interests}

The authors declare that there is no conflict of interests regarding the publication of this paper.

\section{Acknowledgments}

This work was partly supported by the Fundamental Research Funds for the Central Universities No. 27R1410016A.

\section{References}

[1] P. Colli Franzone and E. Magenes, "On the inverse potential problem of electrocardiology," Calcolo, vol. 16, no. 4, pp. 459538,1979 .

[2] C. R. Johnson, "Computational and numerical methods for bioelectric field problems," Critical Reviews in Biomedical Engineering, vol. 25, no. 1, pp. 1-81, 1997.

[3] G. Alessandrini, "Stable determination of a crack from boundary measurements," Proceedings of the Royal Society of Edinburgh: Section A Mathematics, vol. 123, no. 3, pp. 497-516, 1993.

[4] J. Hadamard, Lectures on Cauchy's Problem in Linear Partial Differential Equations, Dover Publications, New York, NY, USA, 1953.

[5] D. N. Hào, T. D. Van, and R. Gorenflo, “Towards the Cauchy problem for the Laplace equation," Partial Differential Equations. Banach Center Publications, vol. 27, no. 1, pp. 111-128, 1992.

[6] J. L. Fleming, "Convergence analysis of a Fourier-based solution method of the Laplace equation for a model of magnetic recording," Mathematical Problems in Engineering, vol. 2008, Article ID 154352, 11 pages, 2008.

[7] L. Eldén and F. Berntsson, "A stability estimate for a Cauchy problem for an elliptic partial differential equation," Inverse Problems, vol. 21, no. 5, pp. 1643-1653, 2005.

[8] X.-L. Feng, L. Eldén, and C.-L. Fu, "A quasi-boundary-value method for the Cauchy problem for elliptic equations with nonhomogeneous Neumann data," Journal of Inverse and IllPosed Problems, vol. 18, no. 6, pp. 617-645, 2010.

[9] C. Vani and A. Avudainayagam, "Regularized solution of the Cauchy problem for the Laplace equation using Meyer wavelets," Mathematical and Computer Modelling, vol. 36, no. 9-10, pp. 1151-1159, 2002.

[10] D. N. Hào, "A mollification method for ill-posed problems," Numerische Mathematik, vol. 68, no. 4, pp. 469-506, 1994.

[11] C.-L. Fu, H.-F. Li, Z. Qian, and X.-T. Xiong, "Fourier regularization method for solving a Cauchy problem for the Laplace equation," Inverse Problems in Science and Engineering, vol. 16, no. 2, pp. 159-169, 2008.

[12] U. Tautenhahn, "Optimal stable solution of Cauchy problems for elliptic equations," Journal for Analysis and Its Applications, vol. 15, no. 4, pp. 961-984, 1996.

[13] U. Tautenhahn, "Optimality for ill-posed problems under general source conditions," Numerical Functional Analysis and Optimization, vol. 19, no. 3-4, pp. 377-398, 1998.

[14] U. Tautenhahn, "Optimal stable approximations for the sideways heat equation," Journal of Inverse and Ill-Posed Problems, vol. 5, no. 3, pp. 287-307, 1997.

[15] U. Tautenhahn and T. Schröter, "On optimal regularization methods for the backward heat equation," Zeitschrift für angewandte Mathematik und Physik, vol. 15, no. 2, pp. 475-493, 1996.

[16] T. Schröter and U. Tautenhahn, "On the optimal regularization methods for solving linear ill-posed problems," Zeitschrift für Analysis und ihre Anwendungen, vol. 13, pp. 697-710, 1994.

[17] G. Vainikko, "On the optimality of methods for ill-posed problems," Zeitschrift für Analysis und ihre Anwendungen, vol. 6, no. 4, pp. 351-362, 1987.

[18] A. A. Melkman and C. A. Micchelli, "Optimal estimation of linear operators in Hilbert spaces from inaccurate data," SIAM Journal on Numerical Analysis, vol. 16, no. 1, pp. 87-105, 1979. 


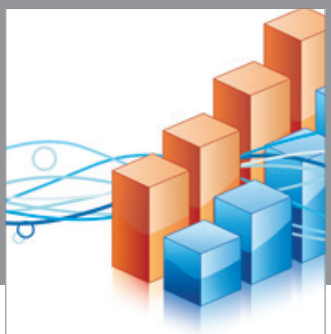

Advances in

Operations Research

vatem alat4

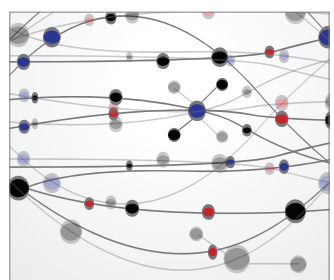

\section{The Scientific} World Journal
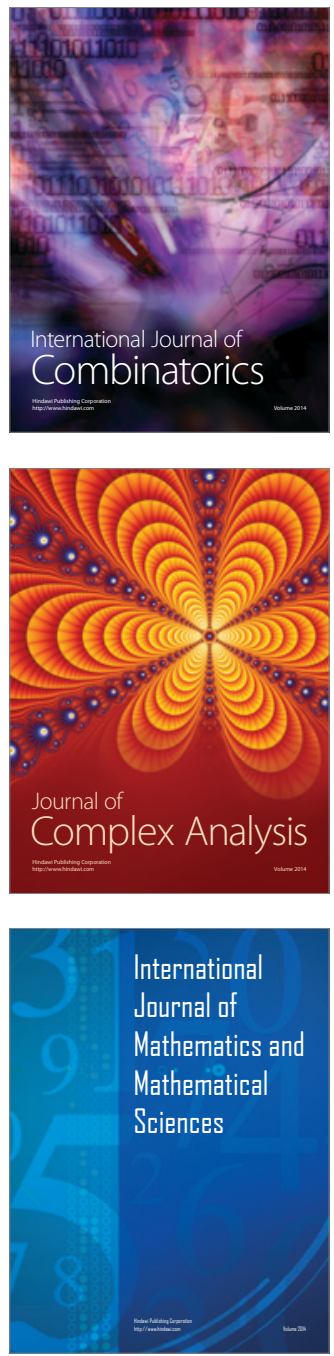
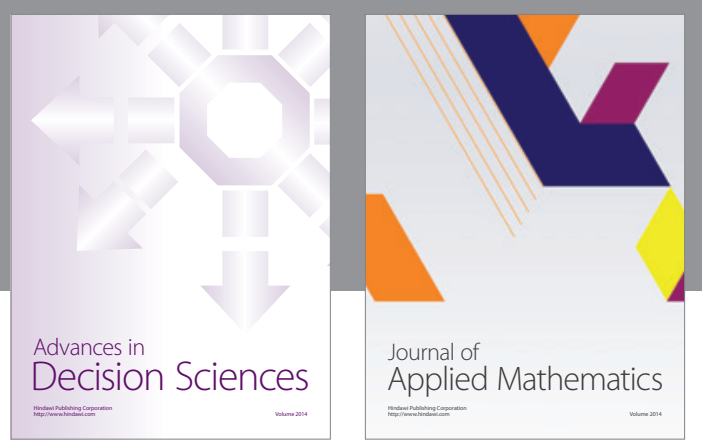

Algebra

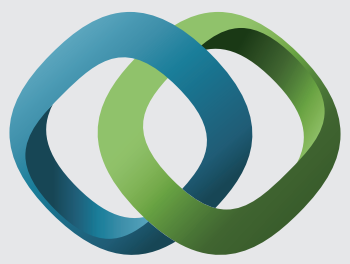

\section{Hindawi}

Submit your manuscripts at

http://www.hindawi.com
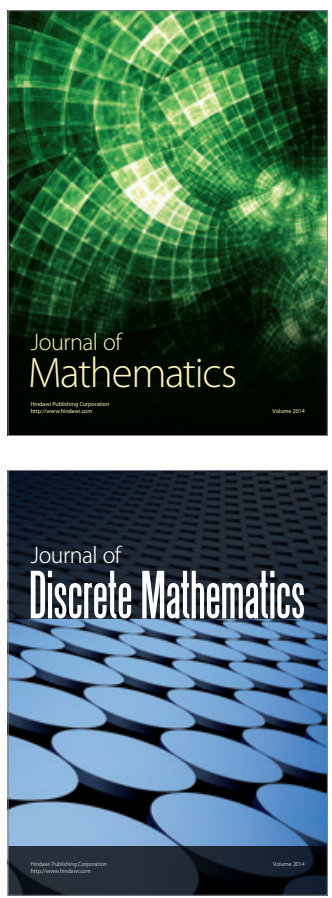

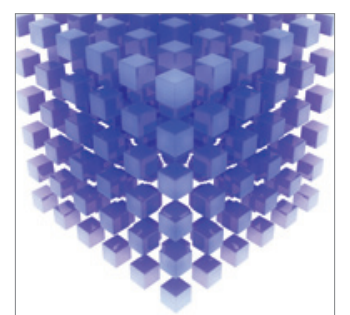

Mathematical Problems in Engineering
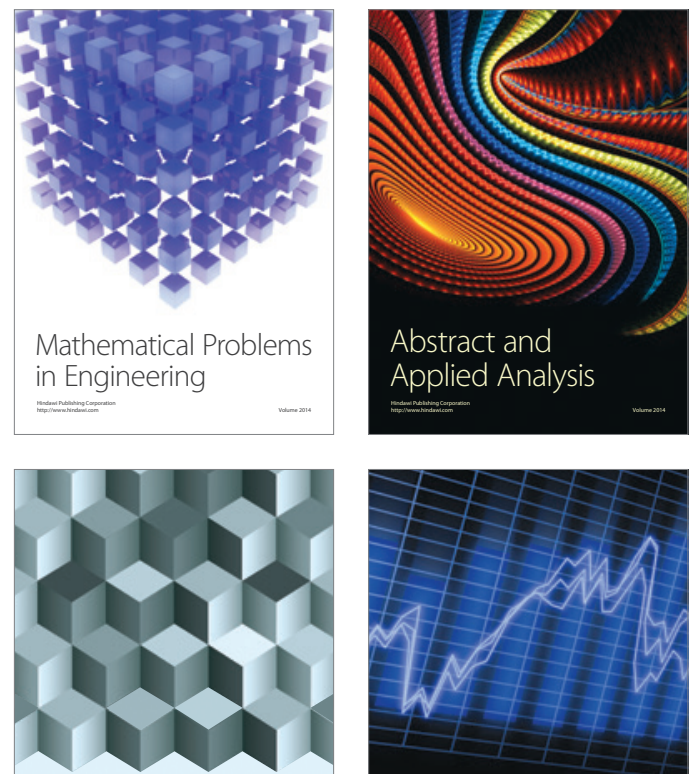

Journal of

Function Spaces

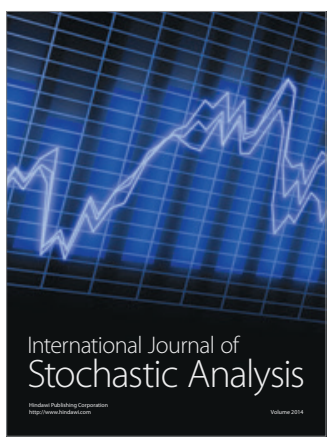

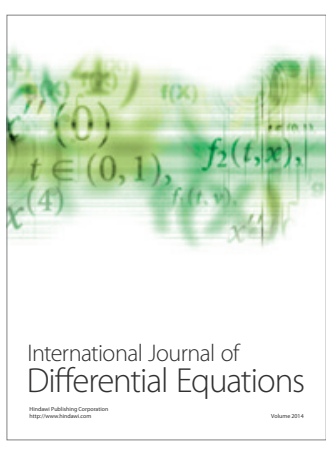
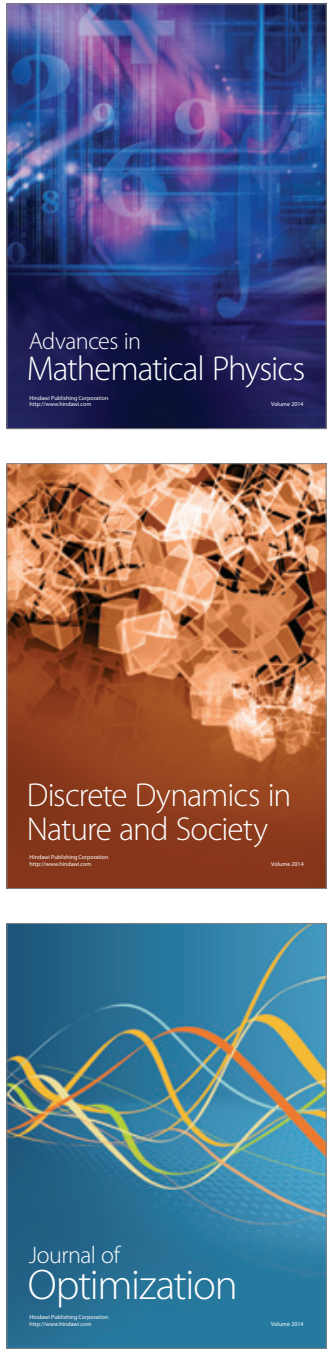